\title{
Using whole immune system characterization (immune profiling) to identify immune biomarkers to determine patient selection, dosing, and efficacy of new immune therapies
}

\author{
Michael Gustafson*, Yi Lin, Dennis Gastineau, lan Parney, Allan Dietz
}

From 30th Annual Meeting and Associated Programs of the Society for Immunotherapy of Cancer (SITC 2015) National Harbor, MD, USA. 4-8 November 2015

\section{Background}

Considerable diversity exists in the immune competency of cancer patients even within patients of the same diagnosis. The relationship between the nature and degree of immune suppression and the responses to different targets of immunotherapy will be the key to the rapid implementation and optimal use of these therapies.

\section{Methods}

We have developed a systems based approach to understand immunosuppression in cancer patients by combining comprehensive whole blood quantitative flow cytometry and classical bioinformatics analyses. Our first generation immune profile analysis identified a unique biomarker consisting of the ratio of CD4+ $\mathrm{T}$ cells to CD14 ${ }^{+}$HLA-DR ${ }^{\mathrm{lo} / \text { neg }}$ monocytes that correlate with survival rates in over 90 patients with glioblastoma, non-Hodgkin lymphoma, renal cell carcinoma or ovarian cancer ${ }^{1}$. Our second generation technology counts over 150 distinct immune phenotypes including over 25 myeloid phenotypes both stimulating and suppressive and many with no known function ${ }^{2}$. There are $10 \mathrm{PD}-1+$ and CTLA-4+ T cell combinations, dozens of other $\mathrm{T}$ cell phenotypes, as well as cell types rarely included in cancer immunology including granulocyte phenotypes.

\section{Results}

As the analysis is technology and disease agnostic, this approach is being used across our immune therapy program. To date, more than 100 individuals have been typed. As an example we present preliminary longitudinal data from newly diagnosed glioblastoma patients enrolled in a Phase I clinical trial $(n=8)$ receiving a dendritic cell vaccine with concurrent standard of care demonstrating immunophenotypic changes during the course of therapy.

\section{Conclusions}

The data collected from these studies will provide valuable insight in the immunological deficits of patients prior to treatment and phenotypes that contribute to positive or negative responses. This methodology will likely improve various aspects of dendritic cell and other cancer immunotherapies through the optimization of the targeting of specific deficits, timing of delivery, and the development of combinatorial approaches.

ClinicalTrials.gov Identifier: NCT01957956

Published: 4 November 2015

\section{References}

. Gustafson MP, Lin Y, LaPlant B, Liwski CJ, Maas ML, League SC, et al: Immune monitoring using the predictive power of immune profiles. J Immunother Cancer 2013, 1:7.

2. Gustafson MP, Lin $Y$, Maas ML, Van Keulen VP, Johnson P, Peikert T, et al: A method for identification and analysis of non-overlapping myeloid immunophenotypes in humans. PloS One 2015, 10(3):e0121546.

doi:10.1186/2051-1426-3-S2-P90

Cite this article as: Gustafson et al:: Using whole immune system characterization (immune profiling) to identify immune biomarkers to determine patient selection, dosing, and efficacy of new immune therapies. Journal for ImmunoTherapy of Cancer 2015 3(Suppl 2):P90.

Mayo Clinic, Rochester, MN, USA 